\title{
Sobre la aplicación de criterios de estabilidad a sistemas mecánicos obtenidos por un método variacional.
}

RAFAEL RODRIGUEZ REBOLLO, Dr. Ingeniero de Caminos

$070 \cdot 8$

sinopsis

Estudiamos la establlidad de estados de verios sistemas mecénicos concretos, blen de sólidos rigldos o blen ldaticos, para colucionss de tlpo exponencial respecto del tempo. Consideramos lo problemas de autovalores correspondientes dichas soluciones obteniendo criterlos de eatabilidad por un metodo variacional. Eatos criterios colnciden con resultados conocidos o aportan otros nuevos.

\section{INTRODUCCION}

En un trabajo anterior [1] se desarrolló un método variacional para la obtención, de forma unificada, de condiciones suficientes de estabilidad de estados de sistemas mecánicos, discretos y continuos. Las ecuaciones variacionales de movimiento, para pequeñas perturbaciones, tenian una estructura formal común frecuente en diversos problemas de estabilidad de sistemas de particulas y de sólidos rigidos y de sistemas continuos.

El método desarrollado es particularmente útil para sistemas discretos de dos o tres dimensiones o para sistemas elásticos monodimensionales. En este caso, la aplicación de métodos aproximados, como el de Garlekin, permite la reducción a un problema discreto en dos o tres dimensiones, suficiente en muchos casos en la práctica.

En el presente trabajo, aplicamos dicho método a varios problemas concretos.

\section{FORMULACION GENERAL}

Hacemos una rápida sintesis del método general.

Consideramos las siguientes ecuaciones variacionales de movimiento:

$$
\overrightarrow{F u}=\vec{u}+2 \hat{E} \vec{u}+\overrightarrow{D u}=\overrightarrow{0}
$$

donde $u_{i}(\vec{x}, t)=0$ representa la configuración respecto a la cual se estudian las propiedades de estabilidad.

$E$ y $\bar{D}$ son operadores lineales diferenciales sobre $\vec{x}$, con coeficientes reales constantes, 0 bien matrices con elementos reales constantes.

Consideramos las soluciones del tipo $\vec{u}=\vec{\eta}=\vec{f}(\vec{x}) e^{\text {iw }}, \omega=\alpha+i \beta, \alpha$ y $\beta$ reales, $\beta \geqslant 0$.

Constituyen un espacio $P$, en el que definimos el producto interno, como es usual:

$$
\overrightarrow{(\eta \eta}, \vec{\xi})=\int_{v(\eta)} \vec{\eta} \cdot \vec{\xi} d V
$$

donde el asterisco indica "complejo conjugado", siendo la integral finita. $V(t)$ es el volumen del sólido para $\vec{u}=\overrightarrow{0}$. 
En el caso general, para $\vec{u}=\vec{\eta}$, la ecuación (1) se escribe:

$$
\vec{\eta}=-\hat{L} \vec{\eta}=-\left[\left(\alpha^{2}-\beta^{2}\right)+2 \alpha \beta \vec{j} \vec{\eta}\right.
$$

donde

$$
\mathcal{C}=\hat{G}^{s}+\hat{H}^{a}, \hat{G}^{s}=\hat{D}^{s}-2 \beta \hat{E}^{s}+2 \alpha_{i} \hat{E}^{a}
$$

y

$$
\hat{A}^{\mathrm{a}}=\bar{D}^{\mathrm{a}}-2 \beta \hat{E}^{\mathrm{a}}+2 \alpha_{i} \hat{E}^{\mathrm{s}}
$$

y las letras "a" y "S" indican antihermitico y hermitico, respectivamente.

Consideramos dos casos para el estudio de las condiciones suficientes de estabilidad.

1. Sobre el sistema actuan fuerzas conservativas y giroscópica exclusivamente: $E=E^{a}$ y $\bar{D}=\hat{D}^{\text {s }}$.

Consideramos la funcional $M=\frac{\overrightarrow{(\eta}, \overrightarrow{F \eta})}{(\vec{\eta}, \vec{\eta})}$. Los valores de $M$ tales que $\delta M=0$, son los autovalores $\lambda_{F}$. La condición de estabilidad es que los valores de $\omega$ soluciones de la ecuación $\lambda_{F}=0$ sean reales.

Condición suficiente de estabilidad es:

$$
\begin{aligned}
& \left.\hat{\lambda}_{F}\right|_{n=(j)^{\prime}}>0 \quad[2] \\
& \omega=\omega^{\prime} \text { tal que }\left.\frac{\partial \hat{\lambda}_{F}}{\partial \omega}\right|_{m=(j)^{\prime}}=0
\end{aligned}
$$

Si el espectro de autovalores está acotado, bastará expresar que el menor de los $\lambda_{F}$ sea positivo.

II. En el caso general, utilizamos la misma funcional $M$, obteniendo en este caso autovalores que son números complejos.

La condición de estabilidad es que $\lambda_{F}^{r}=0$ para $\alpha=\alpha+i \beta, \alpha$ y $\beta$ reales, $\beta \geqslant 0$, tales que $\lambda_{F}^{\prime}=0$. Los valores $\beta>0$ dan estabilidad asintótica. Las letras " $p$ " $\beta$ " indican real e imaginario respectivamente.

Condición suficiente de estabilidad es:

$$
\text { Menor }\left.\lambda_{F}^{r}\right|_{\alpha=\alpha^{\prime}}>0, \alpha=\alpha^{\prime} \text { tal que }\left.\lambda_{F}^{\prime}\right|_{\alpha=\alpha^{\prime}}=0,
$$

$\beta=0$. Son condiciones limites. 


\section{APLICACIONES}

\section{Ejemplo 1}

Un sistema giroscópico de $n$ grados de libertad es tal que la energia cinética es $T=\frac{1}{2} \vec{q}^{T} A \vec{q}$, $\operatorname{con} A=a_{i i}(\vec{q}), i, j=1,2, \ldots, n, a=c t e, y$ la energia potencial, $V=V\left(q_{2}, \ldots, q_{n}\right)$ en el espacio $(\vec{q}, \vec{q} t)$. Definimos la posición de equilibrio aparente 0 dinámico $\left(q_{02}, \ldots, q_{0 n}\right)$ como aquella en que todas las $\frac{\partial V}{\partial q_{i}}(i \geqslant 2)$ son nulas.

En el estado no perturbado existe realmente movimiento del sistema.

Sea un sistema giroscópico con dos coordenadas no cíclicas, en particular una peonza simétrica y hallemos condiciones para la estabilidad de la posición de equilibrio aparente.

En forma general, las ecuaciones variacionales de movimiento en la aproximación lineal son:

$$
\hat{F} \vec{u} \equiv \vec{u}+2 \hat{E}^{\theta} \vec{u}+\hat{D}^{s} \vec{u}=\overrightarrow{0}
$$

$\operatorname{con} E^{a}=\left(\begin{array}{cc}0 & p \\ -\infty & 0\end{array}\right), \rho>0$

$$
D^{s}=\left(\begin{array}{cc}
q 1 & \\
0 & q_{2}
\end{array}\right)
$$

Sea:

$$
\vec{u}=\vec{\eta}=\left(\begin{array}{l}
A_{1} e^{j \varphi_{r}} \\
A_{2} \mathrm{e}^{j / \varphi_{2}}
\end{array}\right) e^{i \omega_{t}}
$$

Resulta:

$$
\begin{gathered}
M=\left(q_{1}-\omega^{2}\right) \frac{A_{1}^{2}}{A_{1}^{2}+A_{2}^{2}}+\left(q_{2}-\omega^{2}\right) \frac{A_{2}^{2}}{A_{1}^{2}+A_{2}^{2}}+ \\
+4 \rho \omega \frac{A_{1} A_{2} \operatorname{sen}\left(\varphi_{1}-\varphi_{2}\right)}{A_{1}^{2}+A_{2}^{2}}
\end{gathered}
$$

De $\delta M=0$, obtenemos:

$$
\begin{aligned}
& A_{2} \delta A_{1}\left[2 A_{1} A_{2}\left(q_{1}-q_{2}\right)+4 p \omega \operatorname{sen}\left(y_{1}-y_{2}\right)\left(A_{2}^{2}-A_{1}^{2}\right)\right]+ \\
& +A_{1} \delta A_{2}\left[-2 A_{1} A_{2}\left(q_{1}-q_{2}\right)-4 p \omega \operatorname{sen}\left(y_{1}-y_{2}\right)\left(A_{1}^{2}-A_{2}^{2}\right)\right]+ \\
& +\left(\delta \varphi_{1}-\delta \varphi_{2}\right) \cos \left(\varphi_{1}-\varphi_{2}\right) A_{1} A_{2}\left(A_{1}^{2}+A_{2}^{2}\right)=0
\end{aligned}
$$

Tomamos $\varphi_{1}-\varphi_{2}=\frac{\pi}{2}$ y tenemos:

$$
A_{1} A_{2}\left(q_{1}-q_{2}\right)+2 p \omega\left(A_{2}^{2}-A_{1}^{2}\right)=0
$$


De esta condición, obtenemos:

$$
A_{2}=m A_{1}, m=\frac{-\left(q_{1}-q_{2}\right) \pm \sqrt{\left(q_{1}-q_{2}\right)^{2}+16 p^{2} \omega^{2}}}{4 p \omega}
$$

y

$$
\lambda_{F}=\frac{q_{1}-\omega^{2}}{1+m^{2}}+\frac{\left(q_{2}-\omega^{2}\right) m^{2}}{1+m^{2}}+4 p \omega \frac{m}{1+m}=\frac{q_{1}+q_{2} m+4 p \omega m}{1+m^{2}}-\omega^{2}
$$

$\lambda_{F}=0$ debe dar las soluciones $\omega$ reales.

De

$$
M=-\omega^{2}+4 p \frac{A_{1} A_{2}}{A_{1}^{2}+A_{2}^{2}}(\omega)+\frac{q_{1} A_{1}^{2}+q_{2} A_{2}^{2}}{A_{1}^{2}+A_{2}^{2}}=0
$$

Obtenemos que:

$$
\omega=2 p \frac{A_{1} A_{2}}{A_{1}^{2}+A_{2}^{2}} \pm \sqrt{\frac{4 p^{2} A_{1}^{2} A_{2}^{2}}{\left(A_{1}^{2}+A_{2}^{2}\right)^{2}}+\frac{q_{1} A^{2}+q_{2} A_{2}^{2}}{A_{1}^{2}+A_{2}^{2}}}
$$

El radicando ha de ser positivo o nulo, lo que conduce a la condición:

$$
q_{1} A_{1}^{4}+\left(4 p^{2}+q_{1}+q_{2}\right) A_{1}^{2} A_{2}^{2}+q_{2} A_{2}^{4} \geqslant 0
$$

1. Es suficiente que sea $q_{1}>0$ y $q_{2}>0$.

2. Si $q_{1}<0\left(0^{\prime} q_{2}<0\right)$, consideramos el signo igual y:

$$
\left(\frac{A_{1}}{A_{2}}\right)^{2}=\frac{-\left(4 p^{2}+q_{1}+q_{2}\right) \pm \sqrt{\left(4 p^{2}+q_{1}+q_{2}\right)^{2}-4 q_{1} q_{2}}}{2 q_{1}}
$$

Ha de ser $q_{1} q_{2}>0$ y

$$
\left(4 p^{2}+q_{1}+q_{2}\right)^{2}>4 q_{1} q_{2}
$$

Importante resultado conocido. Nos dice que la energla potencial tendrá un máximo o un mínimo en la posición de equilibrio aparente, pero no un puerto.

La condición general de estabilidad será:

$$
\begin{gathered}
q_{1} q_{2}>0 \\
\left(4 p^{2}+q_{1}+q_{2}\right)^{2}>4 q_{1} q_{2}
\end{gathered}
$$

resultado conocido.

Estudiamos ahora este problema considerando las condiciones suficientes [2].

En primer lugar, tenemos que:

$$
\begin{gathered}
2 \omega i E^{a}+b^{\mathrm{a}}=\left(\begin{array}{cc}
q_{1} & 2 \omega p i \\
-2 \omega p i & q_{2}
\end{array}\right) \\
\lambda_{F}=\frac{q_{1}+q_{2} \pm \sqrt{\left(q_{1}-q_{2}\right)^{2}+16 \omega^{2} p^{2}}}{2}-\omega^{2}=\lambda_{\theta}-\omega^{2}
\end{gathered}
$$

donde $\lambda_{G}$ es función de $\omega$. 
Supondremos que $q_{1}>q_{2}$.

Utilizaremos la denominación $\lambda_{F}^{+}$y $\lambda_{F}^{-}$según consideremos el signo +0 el - , respectivamente, delante del radical.

Observemos lo siguiente:

$$
\lambda_{F}^{+}>\lambda_{F}^{-}
$$

Tenemos los siguientes extremos de $\lambda_{F}$ :

$$
\frac{\partial \lambda_{F}^{+}--}{\partial \omega}=\frac{ \pm 8 p^{2} \omega}{\sqrt{\left(q_{1}-q_{a}\right)^{2}+16 p^{2} \omega^{2}}}-2 \omega=0
$$

$\lambda_{F}^{+}\left\{\begin{array}{l}\omega_{1}=0 \\ \omega_{2,3}=\frac{ \pm \sqrt{16 p^{4}-\left(q_{1}-q_{2}\right)^{2}}}{4 p}\end{array} \quad \lambda_{F}^{-}\left\{\omega_{1}=0\right.\right.$

y:

$$
\frac{\partial^{2} \lambda_{F}^{+}-}{\partial \omega^{2}}= \pm \frac{8 p^{2}}{\sqrt{\left(q_{1}-q_{2}\right)^{2}+16 p^{2} \omega^{2}}}\left(1-16 \rho^{2} \omega^{2}\right)-2
$$

Comprobamos que,

$$
\omega \rightarrow \pm \infty \Rightarrow \lambda_{F}^{+,-} \rightarrow-\infty
$$

a) Analicemos la curva $\lambda_{F}^{+}$en el plano $\left(\omega, \lambda_{F}^{+}\right)$.

Para $\omega=\omega_{1}=0$, tenemos:

$$
\begin{gathered}
\left.\lambda_{F}\right|_{\omega=0} ^{+}=q_{1} \\
\left.\frac{\partial^{2} \lambda_{F}^{+}}{\partial \omega^{2}}\right|_{\omega=0}=2 \frac{4 p^{2}-\left(q_{1}-q_{2}\right)}{q_{1}-q_{2}}>0
\end{gathered}
$$

si $\omega_{2,3}$ real.

Para $\omega=\omega_{2} \circ \omega_{3}$, se obtiene:

$$
\begin{array}{r}
\left.\lambda_{F}^{+}\right|_{\omega=\omega_{x} \Delta \omega=}=\frac{8 p^{2}\left(2 p^{2}+q_{1}+q_{2}\right)+\left(q_{1}-q_{2}\right)^{2}}{16 p^{2}}= \\
=\frac{\left(4 p^{2}+q_{1}+q_{2}\right)^{2}-4 q_{1} q_{2}}{16 p^{2}} \\
\left.\frac{\partial^{2} \lambda_{F}^{+}}{\partial \omega^{2}}\right|_{\omega=\omega=20 \omega_{0}}=-2 \frac{16 p^{4}-\left(q_{1}-q_{2}\right)^{2}}{8 p^{4}}<0,
\end{array}
$$

si $\omega_{2,3}$ real. 
b) Curva $\lambda_{F}^{-}$.

Para $\omega=\omega_{1}=0$, se obtiene:

$$
\begin{gathered}
\lambda_{F}^{-} \mid \omega=0=q_{2} \\
\left.\frac{\partial^{2} \lambda_{F}^{-}}{\partial \omega^{2}}\right|_{\omega=0}=-2 \frac{4 p^{2}+\left(q_{1}-q_{2}\right)}{q_{1}-q_{2}}<0
\end{gathered}
$$

Para $\omega=0$, la concavidad de $\lambda_{F}^{-}$es de sentido contrario que la de $\lambda_{F}^{+}$, en el caso general. Las condiciones suficientes de estabilidad siguen inmediatamente.

1. Es suficiente que se verifique

$$
q_{2}>0
$$

$y$, por tanto, $q,>0$ también.

2. Son suficientes las condiciones:

$$
q_{1}<0
$$

$y$, por tanto, $q_{2}<0$ también, junto con la desigualdad:

$$
\left(4 p^{2}+q_{1}+q_{2}\right)^{2}>4 q_{1} q_{2}
$$

En resumen, las condiciones suficientes de estabilidad son:

$$
\begin{gathered}
q_{1} q_{2}>0 \\
\left(4 p^{2}+q_{1}+q_{2}\right)^{2}>4 q_{1} q_{2}
\end{gathered}
$$

como conocemos.

Si $q_{1}>0, q_{2}>0$, vemos que se verifican las dos desigualdades: si la energia potencial tiene un minimo en la posición de equilibrio, existe estabilidad cualquiera que sea $p$, como es conocido.

Si $q_{1}<0, q_{2}<0, p$ ha de ser suficientemente grande para que se verifique la 2." condición de estabilidad.

\section{Ejemplo 2}

Consideramos el sistema discreto anterior en el caso particular de una peonza simétrica, para la cual $q_{1}=q_{2}=q$, con los valores:

$$
p=\frac{l_{3} \omega 3}{2 l_{1}}, \quad q= \pm \frac{M g 1}{l_{1}},
$$

con $I_{1}, I_{3} \mathrm{~m}$. de $\mathrm{l}$. principales y 1 distancia del punto fijo al c. de $\mathrm{i}$. de la peonza y 63 la velocidad angular de giro alrededor de su eje.

Para estas condiciones, obtenemos de $\delta M=0$ :

$$
A_{1}= \pm A_{2}
$$


Se tiene:

$$
\lambda_{F}^{+,-}=q \pm 2 p \omega-\omega^{2}=\lambda_{G}-\omega^{2}
$$

en este caso con $\lambda_{G}$ función lineal de $\omega$.

$$
\begin{aligned}
& \lambda_{F}^{+}=0 \Rightarrow \omega=p \pm \sqrt{p^{2}+q} \\
& \lambda_{F}^{-}=0 \Rightarrow \omega=-p \pm \sqrt{p^{2}+q}
\end{aligned}
$$

Condiciones de estabilidad

$$
\rho^{2}+q>0
$$

De otra forma:

$$
\begin{gathered}
\frac{\partial \lambda_{F}^{+}}{\partial \omega}=0 \Rightarrow \omega=p \\
\frac{\partial \lambda_{F}^{-}}{\partial \omega}=0 \Rightarrow \omega=-p \\
\left.\lambda_{F}^{+}\right|_{\omega=p}=p^{2}+q \\
\left.\lambda_{F}^{-}\right|_{\omega=-p}=p^{2}+q
\end{gathered}
$$

y obtenemos las condiciones anteriores:

$$
\rho^{2}+q>0
$$

Observemos que el mismo resultado se obtiene de la condición general del Ejemplo 1; para $q_{1}=q_{2}=q$ :

$$
\left(4 p^{2}+q_{1}+q_{2}\right)^{2}>4 q_{1} q_{2} \Rightarrow p^{2}+q>0 .
$$

Para energia potencial con un minimo en la posición de equilibrio aparente vertical, la condición anterior se verifica siempre, como sabemos.

Si la energía potencial tiene un máximo, $q<0$ y entonces $p$ ha de ser suficientemente grande.

Veamos el significado físico:

$$
p^{2}+q>0 \Rightarrow \frac{l_{3}^{2} \omega_{3}^{2}}{4 l_{1}^{2}}-\frac{M g l}{l_{1}}>0
$$

luego

$$
\beta=l_{3} \omega_{3}>2 \sqrt{M g I_{1}}
$$

En otras palabras, una peonza simétrica, girando alrededor de su eje, es estable respecto a la posición vertical de energia potencial máxima si la velocidad angular $\omega_{3}$ de dicho giro es superior al valor obtenido, para la aproximación lineal. 


\section{Ejemplo 3}

Consideramos el sistema giroscópico del Ejemplo 2, introduciendo fuerzas disipativas.

$$
\hat{F} \vec{u} \equiv \ddot{\vec{u}}+2\left(\hat{E}^{s}+\hat{E}^{a}\right) \dot{\vec{u}}+\hat{D}^{s} \vec{u}=\overrightarrow{0}
$$

con

$$
\begin{aligned}
& \hat{D}^{s}=\left(\begin{array}{ll}
q & 8 \\
0 & 8
\end{array}\right), \hat{E}^{s}=\left(\begin{array}{ll}
s & 0 \\
8 & { }_{a}
\end{array}\right), \hat{E}^{a}=\left(\begin{array}{cc}
0 & p \\
-p & 0
\end{array}\right) \\
& s>0, p>0 . \\
& \text { Para } \vec{u}=\left(\begin{array}{l}
A_{1} e^{\left(\varphi_{1}\right.} \\
A_{2} e^{i \varphi_{2}}
\end{array}\right) e^{(\omega) t}, \omega=\alpha+i \beta
\end{aligned}
$$

Obtenemos:

$$
M=q-\omega^{2}+2 \omega s i+4 p \omega \frac{A_{1} A_{2}}{A_{1}^{2}-A_{2}^{2}} \operatorname{sen}\left(\varphi_{1}-\varphi_{2}\right)
$$

$\delta M=0$ nos $\mathrm{da}:$

$$
\begin{gathered}
4 p \omega A_{1} A_{2}\left(A_{1}^{2}+A_{2}^{2}\right) \cos \left(\varphi_{1}-\varphi_{2}\right) \delta\left(\varphi_{1}-\varphi_{2}\right)+ \\
+4 p \omega \operatorname{sen}\left(\varphi_{1}-\varphi_{2}\right)\left[\left(A_{2}^{2}-A_{1}^{2}\right) A_{1} \delta A_{2}-\left(A_{1}^{2}-A_{2}^{2}\right) A_{2} \delta A_{1}\right]=0
\end{gathered}
$$

Tomamos:

$$
\varphi_{1}-\varphi_{2}=\frac{\pi}{2}
$$

y tenemos:

$$
A_{1}= \pm A_{2}
$$

Por tanto:

$$
\begin{aligned}
& \lambda_{F}^{+}=q+2 p \omega-\omega^{2}+2 \omega s i \\
& \lambda_{F}^{-}=q-2 p \omega-\omega^{2}+2 \omega s i
\end{aligned}
$$

Condición necesaria de estabilidad es que $\lambda_{F}=0$ tenga las soluciones $\omega=\alpha+i \beta$, con $\alpha$ y $\beta$ reales, $\beta \geqslant 0$.

Existe estabilidad asintótica o estabilidad según sea $\beta$ mayor o igual que cero, respectivamente.

Tenemos

$$
\lambda_{F}^{+}{ }^{-}=q \pm 2 p \alpha-\alpha^{2}+\beta^{2}-2 \beta s+i( \pm 2 p \beta+2 s \alpha-2 \alpha \beta)
$$


3.1. Para $\beta=0$, tenemos:

$$
\begin{gathered}
\operatorname{Im} \lambda_{F}^{+,}-=0 \Rightarrow \alpha=0 \\
\text { Re } \lambda_{F}^{+,}-\left.\right|_{\alpha, \beta=0}=q
\end{gathered}
$$

Las $\lambda_{F}^{r}$ con $\beta=0$ son parábolas con la concavidad hacla las $\lambda_{F}^{r}$ negativas:

$$
\begin{gathered}
\text { Re } \left.\lambda_{F}^{+}\right)^{-}=-\alpha^{2} \pm 2 \alpha p+q \\
\text { con un máximo para } \alpha= \pm p
\end{gathered}
$$

Condición suficiente de estabilidad:

$$
q>0
$$

es decir, la energia debe tener un mínimo en la posición de equilibrio aparente.

Observemos que para $q>0$, el punto $(0,0,0)$ del triedro trirrectángulo $\left(\alpha, \beta, \lambda_{F}^{r}\right)$ está comprendido entre las dos intersecciones de $\lambda_{F}^{r+}$ y $\lambda_{F}^{r-}$ con el eje $\beta=0$. $\lambda_{F}^{r}=0$. En cambio, para $q<0$, el origen no está comprendido entre las citadas intersecciones.

Analicemos la intensidad de la peonza cuando la energía potencial tiene un máximo, $q<0$, de la siguiente manera:

$$
\lambda_{F}=q \pm 2 p \omega-\omega^{2}+2 \omega s i
$$

$\lambda_{F}=0$ debe dar las $\omega=\alpha+i \beta$, con $\alpha$ y $\beta$ reales y $\beta>0$ para la estabilidad asintótica.

Por tanto, resolvemos las ecuaciones:

$$
\begin{gathered}
\lambda_{F}^{+}=q-\omega^{2}+2 \omega(p-s i)=0 \\
\lambda_{F}^{-}=q-\omega^{2}+2 \omega(p+s i)=0 \\
\omega^{+}=-(p-s i) \pm(a-b i)=\alpha_{1}+i \beta_{1} \\
\omega^{-}=p+s i \pm(a+b i)=\alpha_{2}+i \beta_{2}
\end{gathered}
$$

donde

$$
\begin{aligned}
& a=\sqrt{\frac{\sqrt{\left(p^{2}+q-s^{2}\right)^{2}+4 p^{2} s^{2}+\left(p^{2}+q-s^{2}\right)}}{2}} \\
& b \sqrt{\frac{\sqrt{\left(p^{2}+q-s^{2}\right)^{2}+4 p^{2} s^{2}-\left(p^{2}+q-s^{2}\right)}}{2}}
\end{aligned}
$$

Calculemos el término imaginario:

$$
b^{2}-s^{2}=\frac{\sqrt{\left(p^{2}+q+s^{2}\right)^{2}-4 q s^{2}}-\left(p^{2}+q+s^{2}\right)}{2}
$$

Si $q>0$, entonces $s \pm b$ es mayor que cero y existe estabilidad asintótica.

Si $q<0$, suponiendo $p^{2}+q>0$ o más aún $p^{2}+q-s^{2}>0$, entonces $s+b$ es positiva, pero $s-b$ es negativa y existen soluciones crecientes exponencialmente. Para $q<0$ existe inestabilidad.

Las fuerzas giroscópicas con disipación dan inestabilidad, aunque se cumpla las condiciones suficientes de estabilidad sin fuerzas disipativas, en el caso de que la energia potencial en la posición de equilibrio aparente tenga un máximo. $Y$ aun suponiendo una restricción mayor $p^{2}+$ $+q-s^{2}>0$ que en aquel caso. 
3.2. Veamos el problema de forma general.

$$
\begin{gathered}
\lambda_{F}^{r}=q \pm 2 p \alpha-\alpha^{2}+\beta^{2}-2 \beta s \\
\lambda_{F}^{\prime}= \pm 2 p \beta+2 s \alpha-2 \alpha \beta
\end{gathered}
$$

Ha de ser $\lambda_{F}^{r}=\lambda_{F}^{\prime}=0$ para $\alpha$ y $\beta$ reales, $\beta \geqslant 0$. El valor positivo de $\beta$ corresponde a estabilidad asintótica.

La condición que resulta operando es $q>0$, para $\beta>0$. La condición, ya obtenida en 3.1., corresponde a estabilidad asintótica.

A continuación se representan gráficamente la curva $\lambda_{F}^{j}=0$ en el plano $(\alpha, \beta)$ y la curva $\lambda_{F}^{r}=0$, en este caso para $\beta=0$, en el plano $\left(\alpha, \lambda_{F}^{r}\right)$ y se deducen geométricamente las condiciones suficientes de estabilidad.

En primer lugar, estudiamos:

$$
\lambda_{F}^{r,+}=q+2 p \alpha-\alpha^{2}+\beta^{2}-2 \beta s
$$
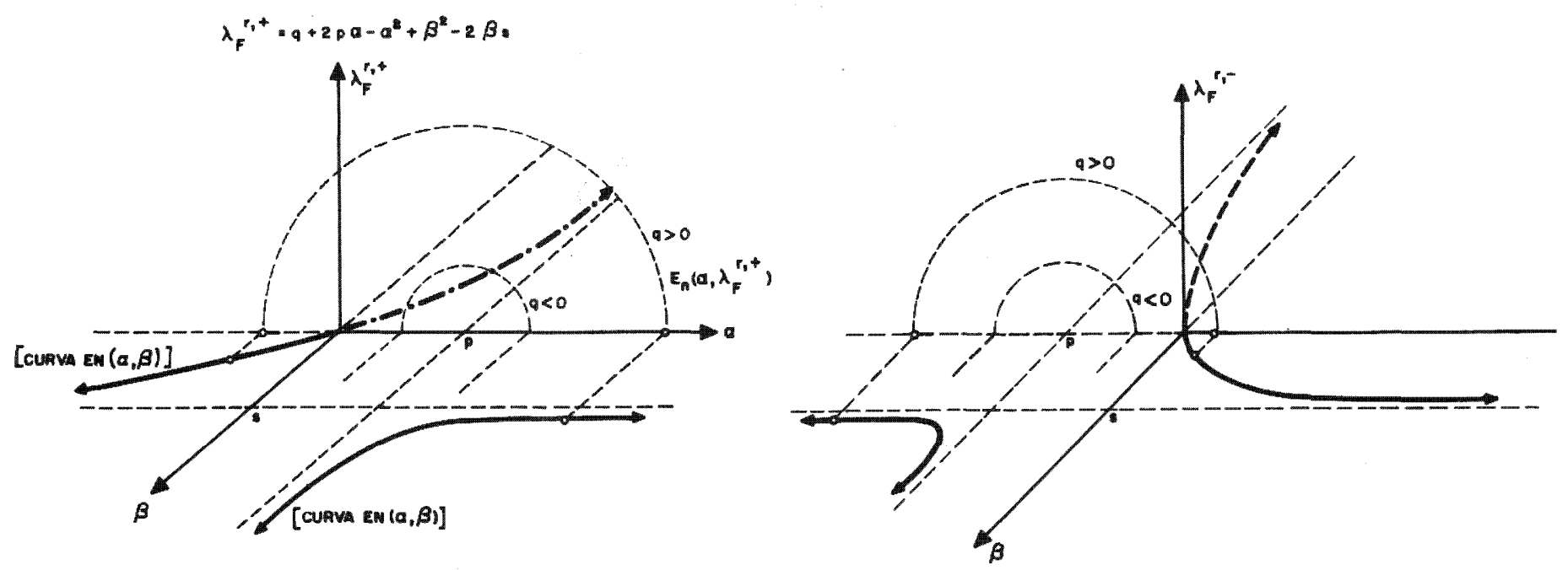

Vemos que sólo las intersecciones de la curva $\lambda_{F}^{r} \pm\left.\right|_{p=0, q>0}$ con el eje de las $\alpha$ contienen al origen. Existe estabilidad, al menos, para $q>0$.

Considerando las proyecciones de dichas intersecciones sobre la curva $\lambda_{F}^{i}$, podemos deducir:

Si $q>0$, Estabilidad asintótica.

Si $q<0$, Inestabilidad.

Sea ahora $\lambda_{f}^{r-}$. De forma análoga, vemos que:

Si $q>0$, Estabilidad asintótica.

Si $q<0$, Inestabilidad. 


\section{Ejemplo 4}

Consideramos una columna elástica, articulada en sus dos extremos y sometida a la acción de una carga vertical constante en dirección e intensidad, $q$ por unidad de longitud, en particular su propio peso.

Sea 1 la longitud total, $E$ el módulo de elasticidad e $l$ el momento de inercia de la sección transversal, constantes. Entonces las ecuaciones de movimiento se escribe:

$$
\begin{gathered}
\hat{F} u \equiv E I u_{x x x x}+q(1-x) u_{x x}-q u_{x}+\ddot{u}=0 \\
\operatorname{con} u(0)=u(1)=u_{x x}(0)=u_{x x}(1)=0
\end{gathered}
$$

Para $u=\eta=f(x) e^{j \omega_{t}}$

$$
\begin{aligned}
& \frac{\overrightarrow{(\vec{F} F \vec{u})}}{\overrightarrow{(u \vec{u})}}=\frac{E l \int_{a}^{1} u u_{x x x x} d x+q \int_{a}^{1}\left[(1-x) u_{x x}-u_{x}\right] u d x}{\int_{0}^{1} u^{x} u d x}+\ddot{u}= \\
& =-\omega^{2}+\frac{E l \int_{0}^{1} f_{x x} f^{x x} d x-q \int_{0}^{1}(1-x) f_{x} f_{x} d x}{\int_{0}^{1} f^{x f} d x}=\lambda_{G}-\omega^{2}
\end{aligned}
$$

Condiciones de estabilidad:

$$
\left.\lambda_{F}\right|_{\omega=0}=\lambda^{a}>0
$$

Pero observamos lo siguiente:

$$
\int_{0}^{1} f_{x x}^{x} f_{x x} d x \geqslant \lambda_{F} \int_{0}^{1}(1-x) f_{x}^{z f} f_{x} d x
$$

$\lambda_{R}$ cociente de Rayleigh conocido:

$$
\lambda_{R} \leqslant \frac{\int_{0}^{1} f_{x x} d x x}{\int_{0 x x}^{1}\left\{-f^{x}\left[(1-x) f_{x}\right]_{x}\right\} d x}
$$

Por tanto:

$$
\begin{aligned}
& \lambda_{G} \geqslant \frac{E\left(\lambda_{A} \int_{0}^{1}(1-x) f_{x}^{x_{x}} f_{x} d x-q \int_{0}^{1}(1-x) f_{x}^{x} f_{x} d x\right.}{\int_{0}^{1} f x f d x}= \\
& =m\left(E / \lambda_{R}-q\right) \frac{\int_{0}^{1}(1-x) f_{x}^{x} f_{x} d x-q \int_{0}^{1}(1-x) f_{x}^{x} f_{x} d x}{\int_{0}^{1} f^{x} f d x}=
\end{aligned}
$$




$$
=m\left(E \mid A_{R}-q\right) \frac{\int_{0}^{1} f_{x}^{x} f_{x} d x}{\int_{0}^{l} f x f d x}, d o n d e 0<m<1
$$

tal que:

$$
\int_{0}^{1}(1-x) f_{x}^{f_{x}} f d x=m \int_{0}^{1} f_{x}^{x} f_{x} d x
$$

por el teorema del valor medio.

La condición de estabilidad será:

$$
\begin{aligned}
& E / \lambda_{A}-q>0 \\
& q_{\text {eritica }}=E / \lambda_{\text {A }}
\end{aligned}
$$

resultado conocido y obtenido aqui de manera más simple.

Consideremos ahora una solución aproximada:

$$
u=\left[\rho_{1} f_{1}(x)+\rho_{2} f_{2}(x)\right] e^{k \omega t}=\left(\rho_{1} \operatorname{sen} \frac{\pi x}{1}+\rho_{2} \operatorname{sen} \frac{2 \pi x}{1}\right) e^{i \omega t}
$$

limitándonos a dos términos, como es usual en la práctica.

Para la funcional $M$, tendremos:

Para $\omega=0$

$$
\begin{gathered}
M=\frac{A_{1} \rho_{1}^{2}+A_{2} \rho_{1} \rho_{2}+A_{3} \rho_{2}^{2}}{\rho_{1}^{2}+\rho_{2}^{2}}-\omega^{2} q \\
\operatorname{con} A_{1}=\frac{\alpha \pi^{4}}{2 l^{3}}-\frac{q \pi^{2}}{4}-q_{n}^{2} \\
A_{2}=-\frac{40}{9} q \\
A_{3}=\frac{8 \alpha \pi 4}{1^{3}}-q \pi^{2} \\
\delta M=0 \Rightarrow\left(A_{1}-A_{3}\right) \rho_{1} \rho_{2}^{2}+\frac{A_{2}}{2}\left(r_{2}^{2}-\rho_{1}^{2}=0\right.
\end{gathered}
$$

menor $\left.\lambda_{F}\right|_{\omega=0}>0$

nos da las condiciones de estabilidad, que resultan:

$$
\text { menor } \frac{A_{1}+A_{3} \pm \sqrt{\left(A_{1}-A_{3}\right)^{2}-A_{2}^{2}}}{2}>0
$$

luego: $\left(A_{1}+A_{3}\right)^{2}>\left(A_{1}-A_{3}\right)^{2}-A_{2}^{2}$ que equivale a:

$$
\begin{gathered}
4 A_{1} A_{3}>A_{2}^{2} \\
\left(\frac{\alpha n^{4}}{2 l^{3}}-q \frac{n^{2}}{4}\right)\left(\frac{8 \alpha n^{4}}{l^{3}}-q n^{2}\right)>\left(\frac{20}{9} q\right)^{2}
\end{gathered}
$$

El signo igual nos da la $q_{\text {crit. }}$, carga crítica:

$$
q_{\text {crit. }}=18,58 \frac{\alpha}{13}
$$

resultado conocido. 


\section{Ejemplo 5}

Consideremos la estabilidad de una posición de equilibrio de un sólido elástico, una placa delgada, uniforme, de longitud 1 en la dirección de las $x$, apoyada en $x=0, x=1$, sometida a la acción de un flujo de fluido, según $x$, por una de las cargas.

En la dirección normal a $x$, la longitud es infinita.

Este problema es muy conocido y su interés aqui estriba en que la ecuación de movimiento perturbado alrededor de la posición de equilibrio horizontal aparece en problemas de placas y columnas, útiles en estructuras.

Tenemos:

$$
F u \equiv \mu \ddot{u}+\dot{u}+a u_{x x x x}-b u_{x x}+M u_{x}=0
$$

con $\mu$, a y $M$ cantidades positivas.

Las condiciones de contorno son:

$$
u=u_{x x}=0 \text {, en } x=0,1, t \geqslant 0 .
$$

Tomemos: $u=\eta=f(x) e^{i \omega t}, \omega=\alpha+i \beta$

Si calculamos $\delta M=0$, obtenemos el siguiente resultado para los autovalores

$$
\begin{gathered}
-\mu \omega^{2} \eta+i \omega \eta+a \eta_{x x x x}-b \eta_{x x}+M \eta^{x}=\lambda_{F} \eta \\
-\mu \omega^{2} \eta^{x}+i \omega \eta^{x}+a \eta_{x x x x}^{x}-b \eta_{x x}^{x}-M \eta_{x}^{x}=\lambda_{F} \eta^{x}
\end{gathered}
$$

De donde:

$$
\lambda_{F}=-\mu \omega^{2} f+a f^{f x \times x}-b f^{x x}+i \omega f+M f^{x}
$$

Las condiciones de contorno son:

$$
f+f^{x}=\left(f+f^{x}\right)_{x x}=0, x=0,1
$$

Si tomamos $f=-i \theta^{i k x}$, con $k=\pi$, obtenemos:

$$
\lambda_{F}=-\mu\left(\alpha^{2}-\beta^{2}\right)-\beta+a \pi^{4}+b \pi^{2}+i(\alpha-2 \alpha \beta+k M)
$$

5.1. Si consideramos $\beta=0$, tenemos:

$$
\begin{gathered}
\lambda_{F}^{\prime}=0 \Rightarrow \alpha=-\pi M \\
\left.\lambda_{F}^{r}\right|_{\substack{\beta=0 \\
\alpha=\infty \pi M}}=-\mu \pi^{2} M^{2}+a \pi^{\alpha}+b \pi^{2}
\end{gathered}
$$

Condición suficiente de estabilidad:

$$
M^{2}<\frac{a \pi^{2}+b}{\mu}
$$

como es conocido.

De otra forma, se tiene que $\lambda_{\bar{F}=0}^{r}$ para $\beta=0$ es una parábola con un máximo para $\alpha=0$. Ha de ser $a \pi^{2}+b>0$.

De $\lambda_{F}^{\prime}=0$, obtenemos $\alpha=-\pi M$ para $\beta=0$ y este punto sobre el eje $\alpha$ ha de ser interior al segmento comprendido entre las intersecciones de dicha parábola con dicho eje:

como antes.

$$
M^{2}<\frac{a \pi^{2}+b}{\mu}
$$


5.2. En el caso general, se tiene que

$$
\begin{gathered}
\lambda_{F}^{\prime}=0 \Rightarrow \alpha=\frac{\pi M}{2 \beta-1} \\
\lambda_{\alpha=\frac{\pi M}{2 \beta-1}}^{\prime}=0 \Rightarrow 4 \mu \beta^{4}-4(1+\mu) \beta^{3}+\left(4+4 a \pi^{4}+\right. \\
\left.+4 b \pi^{2}+\mu\right) \beta^{2}-\left(4 a \pi^{4}+4 b \pi^{2}+1\right) \beta+\left(-\mu \pi^{2} M^{2}+a \pi^{4}+b \pi^{2}\right)=0
\end{gathered}
$$

y esta ecuación nos debe dar las $\beta$ reales y positivas.

Resultan las condiciones suficientes:

$$
\begin{gathered}
a \pi^{2}+b>0 \\
-\mu M^{2}+a \pi^{2}+b>0
\end{gathered}
$$

coincidiendo con el resultado de 5.1, que vemos es válido para estabilidad asintótica.

Representación gráfica

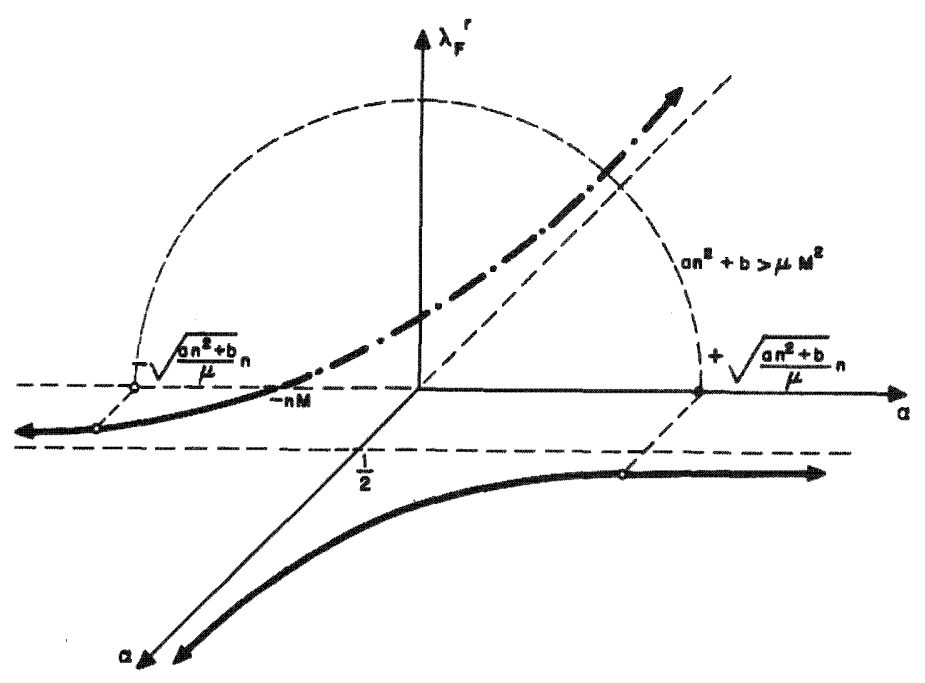

Para $\beta=0$ :

$$
\begin{gathered}
-\mu \alpha^{2}+a \pi^{4}+b \pi^{2}=0 \\
\alpha= \pm \sqrt{\frac{a \pi^{4}+b \pi^{2}}{\mu}}= \pm \pi \sqrt{\frac{a \pi^{2}+b}{\mu}} \\
\text { Ha de ser }-\sqrt{\frac{a \pi^{2}+b}{\mu}} \pi<-\pi M \Rightarrow \mu M^{2}<a \pi^{2}+b
\end{gathered}
$$

para que sea $\beta>0$.

78

(c) Consejo Superior de Investigaciones Científicas

Licencia Creative Commons 3.0 España (by-nc)

http://informesdelaconstruccion.revistas.csic.es 


\section{Ejemplo 6}

Analizamos a continuación la estabilidad de una barra elástica de longitud 1 , cuya ecuación variacional de movimiento, respecto a una posición de equilibrio, se escribe:

$$
\alpha u_{x x x x}+S u_{x x}+Q \dot{u}^{x}+k_{1} u+k_{2} u_{x}+\beta \dot{u}+\ddot{u}=0
$$

con las condiciones de contorno:

$$
u=u_{x x}=0, x=0,1
$$

Suponemos que los coeficientes son constantes, $\operatorname{con} \alpha$ y $\beta$ positivos. El tercer término corresponde a fuerzas giroscópicas producidas por conducción de fluidos. El quinto término representa un caso de fuerzas que varian con la deformación del sólido ("follower forces»). Los restantes términos tienen el significado usual.

Esta ecuación tiene una forma muy general, introduciéndose los nuevos términos $3 .^{\circ}$ y $5 .^{\circ}$ a las ecuaciones de movimiento usuales.

Sean las soluciones $u=\eta=f(x) e^{i \omega_{t}}, \omega=\omega_{r}+i \omega_{1}$.

Operando como en el ejemplo 5, obtenemos que

$$
\lambda_{F}=-\omega^{2 f}+\alpha f_{x x x x}+S f_{x x}+i \omega Q f_{x}+k f f+i \omega f+k_{z} f_{x}
$$

Si consideramos las soluciones $\eta=f(x) e^{i \omega t}=-i e^{i k x} \theta^{i \omega t}, k=\pi$, que representa un caso muy sencillo, se obtiene que:

$$
\begin{gathered}
\lambda_{f}^{r}=-\left(\omega_{r}^{2}-\omega_{2}^{2}\right)-\omega_{2}+\alpha \pi^{4}-S \pi^{2}+k_{1}-\pi \omega_{r} Q \\
\lambda_{F}^{i}=-2 \omega_{r} \omega_{i}+\omega_{r}+\pi k_{2}-\pi \omega_{1} Q
\end{gathered}
$$

6.1. Para $\omega_{l}=0$, se tiene que

$$
\begin{gathered}
\lambda_{F}^{\prime}=0 \Rightarrow \omega_{r}=-\pi k_{2} \\
\left.\lambda_{F}^{r}\right|_{\substack{\omega,=0 \\
\omega, m=-n k_{2}}}=\pi^{2} k_{2}^{2}+\alpha \pi^{4}-S \pi^{2}+k_{1}+\pi^{2} k_{2} Q
\end{gathered}
$$

Condición suficiente de estabilidad:

$$
\alpha \pi^{4}-S \pi^{2}+k_{1}+\pi^{2} k_{2}\left(Q-k_{2}\right)>0
$$

Observemos que la parábola $\left.\lambda_{F}^{r}\right|_{\omega_{1}=0}$ contenida en el plano $\left(\omega_{r}, \lambda_{F}^{r}\right)$ posee un máximo para $\omega_{r}=-\pi \frac{Q}{2}$. Consistente con aquella condición suficiente, ha de ser

$$
\begin{gathered}
\left.\lambda_{F}^{r}\right|_{\omega_{i}=0}=\alpha \pi^{4}-S \pi^{2}+k_{1}+\frac{\pi^{2} Q^{2}}{4}>0 \\
\omega=-\pi \frac{Q}{2}
\end{gathered}
$$

Visto de otra forma, calculemos los puntos de intersección de $\left.\lambda_{F}^{r}\right|_{\omega_{i}=0}$ con el eje $\omega_{r}$. Se tiene que:

$$
\omega_{r}=-\frac{\pi Q}{2} \pm \sqrt{\alpha \pi^{4}-S \pi^{2}+k_{1}+\frac{\pi^{2} Q^{2}}{4}}
$$


La condición de estabilidad será que el punto $\omega_{p}=-\pi k_{2}$ esté comprendido entre las intersecciones de la parábola con el eje $\omega_{r}$ lo que se reduce a la condición suficiente obtenida al comienzo de este apartado.

6.2. En el caso general, se tiene que:

$$
\lambda_{F}^{\prime}=0 \Rightarrow \omega_{1}=\frac{\omega_{r}+\pi k_{2}}{2 \omega_{r}+\pi Q}, \omega_{r}=\frac{\pi \omega_{1} Q-\pi k_{2}}{1-2 \omega_{1}}
$$

Sustituyendo $\omega_{r}$ en $\lambda_{F}^{r}=0$, el resultado es:

$$
\lambda_{F}^{r}=\frac{4 \omega_{1}^{2}-8 \omega_{1}^{3}+\left(\pi^{2} Q^{2}+5\right) \omega_{1}^{2}-\left(\pi^{2} Q^{2}+1\right) \omega_{1}+\alpha \pi^{4}-S \pi^{2}+k_{1}+\pi^{2} k_{a}\left(Q-k_{2}\right)}{\left(1-2 \omega_{1}\right)^{2}}=0
$$

Obtenemos la misma condición suficiente para estabilidad asintótica.

Representación gráfica

Para $k_{2}>0, Q>0$ resulta la figura siguiente:

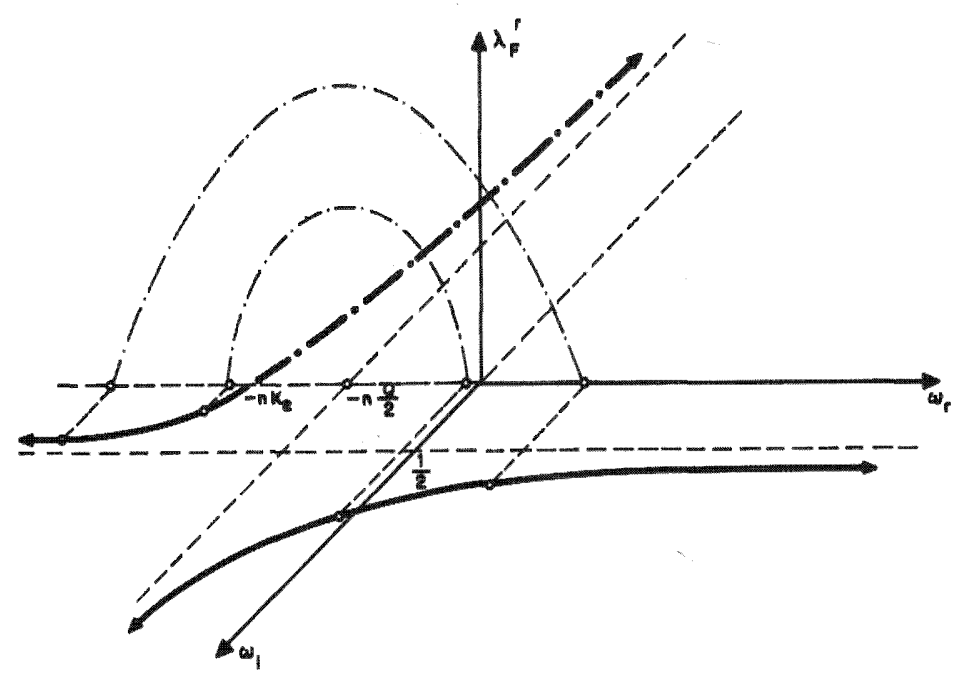

\section{Referenclas}

1. R. Rodriguez Rebollo: Sobre condiciones suficientes de estabilidad para sistemas mecánicos sometidos a fuerzas dependientes de la velocidad por un método variacional. Para ser publicado.

2. L. A. Pars: A treatise on analytical dynamics. Heinemann (1968).

\section{résumé}

Sur l'application de critères de stabilité à des systèmes mécaniques obtenus par une méthode varlationnelle

Rafael Rodriguez Rebollo, Dr, Ingenieur des Ponts et Chaussées

Nous étudions la stabillté d'états de differents systermes mécaniques concrets, solides rigides ou blastlques, pour dos solutions figides ou blastiques, pour des solutions
de type exponentlel par rapport au temps. Nous considerons los problemes d'autova leurs correspondants a cess solutions obte nant des eritéres de stablilie par une máthode varlationnelle. Ces criteres coincident avec des résultats connus at en apportent d’autres nouveaux.

\section{summary}

On the application of stability criteria to mechanical systems obtained through a variational method

Rafal Rodrigua Aebollo, Dr. in Civil Engineering

Studied here is the stablilty state of several specitic mechanical systems, both rigid and elastic, for exponential type solution against time. Considered are the self-value problems of such solutions, obtaining stability criteria by means of varlational method. These erterls elther agree with other already known results, or bring out new ones.

\section{zusammenfassung}

Ueber die anwendung von bastaendigkeitskriterien auf mechanische systeme, die nach iner variationsmethode erhalten wurden

Dr. Rafael Rodriguez Rebollo, Bauingenieur Wir studieren die Beständigkeit der Zus. tănde verschledener konkreter mechanischer Systeme aus starren oder flexiblen Fests. toffen für Lossungen darstellender Art in Hin. sicht aut die Zelt. Wir betrachten die die dlesen Lösungen entsprechenden Selbstwertprobleme und erhalten Beständigkeltskrlto rien nach der Variatlonsmethode. Dlese Krlterien stimmen mit den bekannten Ergab. nissen uberein und verschaffen ausserdem neue. 